\title{
Severe Acute Respiratory Syndrome Coronavirus 2 Induced Focal Segmental Glomerulosclerosis
}

\author{
Is-haq O. Malik ${ }^{1}$, Nida Ladiwala ${ }^{1}$, Siddharth Chinta ${ }^{1}$, Muhammad Khan ${ }^{1}$, Komal Patel $^{1}$ \\ 1. Internal Medicine, BronxCare Health System, Bronx, USA
}

Corresponding author: Is-haq O. Malik, imalik@bronxcare.org

\begin{abstract}
Focal segmental glomerulosclerosis (FSGS) is a common cause of nephrotic syndrome affecting adults and children. Collapsing focal segmental glomerulosclerosis (FSGS), one of five histologic variants of FSGS is described as segmental or global collapse and sclerosis of the glomerular tufts and has been frequently associated with human immunodeficiency virus-associated nephropathy (HIVAN). Its association with other viral and non-viral causes, medications and other disease states has since been established. Due to its resistance to therapy, rapid progression to end-stage renal disease (ESRD) and overall poorer prognosis, identification with electron microscopy examination of the kidney biopsy sample is required during evaluation.
\end{abstract}

Categories: Pathology, Infectious Disease, Nephrology

Keywords: sars-cov-2, covid-19, collapsing fsgs, fsgs, acute kidney injury, proteinuria, esrd, apol1 gene, severe acute respiratory syndrome coronavirus 2 , focal segmental glomerulosclerosis

\section{Introduction}

The novel severe acute respiratory syndrome coronavirus 2 (SARS-CoV-2) has spread globally, with approximately 11 million affected patients and upwards of 500,000 deaths at the time of writing. The disease spread through respiratory droplets and via contact causing pneumonia with multi-organ disease. Though the pathophysiology of coronavirus disease 2019 (COVID-19) causing multi-organ failure has not been fully understood, the interaction between angiotensin-converting enzyme 2 (ACE-2) and SARS family of viruses has been identified as a factor facilitating the infectivity of the virus. Given the abundance of ACE-2 receptors in lungs, pneumonia, and respiratory failure are predominant presentations in symptomatic patients. Initial reports showed acute kidney injury in up to $9 \%$ of patients with COVID-19 with acute tubular injury as the underlying pathophysiological mechanism [1]. However, subsequent studies noted up to $15 \%$ prevalence of kidney injury, with hematuria and proteinuria observed at $26 \%$ and $44 \%$ respectively [2-4]. This paved the way to pursue a kidney biopsy to understand and categorize the mechanism of injury. Presence of chronic kidney disease at baseline has shown to increase mortality risk in patients with COVID-19. We, therefore, present a patient with COVID-19 who developed acute renal failure caused by biopsy-proven collapsing focal segmental glomerulosclerosis (FSGS).

Review began 09/29/2020 Review ended 10/08/2020 Published 10/11/2020

() Copyright 2020 Malik et al. This is an open access article distributed under the terms of the Creative Commons Attribution License CC-BY 4.0., which permits unrestricted use, distribution, and reproduction in any medium, provided the original author and source are credited.

\section{Case Presentation}

We present a 57-year-old African-American male patient who was initially admitted in April with dizziness, diarrhea and hypoxia. The patient had additional symptoms of lymphopenia, elevated inflammatory markers (Table 1) and bilateral patchy infiltrates on chest X-ray (Figure 1), which supported the diagnosis of coronavirus disease 2019 commonly referred to as COVID-19. Polymerase chain reaction (PCR) confirmed severe acute respiratory syndrome coronavirus 2 (SARS-CoV-2) infection in the patient. 


\section{Cureus}

\begin{tabular}{llll}
\hline Laboratory Results on admission & & \\
WBC Count & $7.4(4.8-10.8 \mathrm{k} / \mathrm{ul})$ & Albumin & $3.4(3.4-4.8 \mathrm{~g} / \mathrm{dl})$ \\
Hemoglobin & $17.9(12.0-16.0 \mathrm{~g} / \mathrm{dl})$ & Bilirubin, Total & $0.3(0.2-1.2 \mathrm{mg} / \mathrm{dL})$ \\
Hematocrit & $52.9(42.0-51.0 \%)$ & Bilirubin, Direct - Conjated & $0.2(0.0-0.3 \mathrm{mg} / \mathrm{dL})$ \\
Platelet & $221(150-400 \mathrm{k} / \mathrm{ul})$ & Alkaline Phosphatase & $93(56-119 \mathrm{unit} / \mathrm{L})$ \\
Neutrophil \% & $76.7(40.0-70.0 \%)$ & AST & $93(9-48 \mathrm{unit} / \mathrm{L})$ \\
Lymphocyte \% & $16.8(20.0-50.0 \%)$ & ALT & $47(5-40 \mathrm{unit} / \mathrm{L})$ \\
& $134(135-145 \mathrm{mEq} / \mathrm{L})$ & Total Protein & $7.0(6.0-8.5 \mathrm{~g} / \mathrm{dl})$ \\
Sodium & & LDH & $642(100-190 \mathrm{unit} / \mathrm{L})$ \\
Potassium & $5.8(3.5-5.0 \mathrm{mEq} / \mathrm{L})$ & & $139.80(<=5.00-\mathrm{mg} / \mathrm{L})$ \\
Bicarbonate & $18(24-30 \mathrm{mEq} / \mathrm{L})$ & C Reactive Protein & \\
Chloride & $96(98-108 \mathrm{mEq} / \mathrm{L})$ & & $658(0-230 \mathrm{ng} / \mathrm{mL})$ \\
Glucose & $116(70-120 \mathrm{mg} / \mathrm{dL})$ & D-Dimer Assay & \\
Blood Urea Nitrogen & $27(8-26 \mathrm{mg} / \mathrm{dL})$ & & $1910(13-150 \mathrm{ng} / \mathrm{mL})$ \\
Creatinine & $2.0(0.5-1.5 \mathrm{mg} / \mathrm{dL})$ & Ferritin & \\
Calcium & $8.0(8.5-10.5 \mathrm{mg} / \mathrm{dL})$ & & \\
& & &
\end{tabular}

\section{TABLE 1: Laboratory Results on Admission}

WBC- White Blood Cells; AST- Aspartate Aminotransferase; ALT- Alanine aminotransferase; LDH- Lactate Dehydrogenase

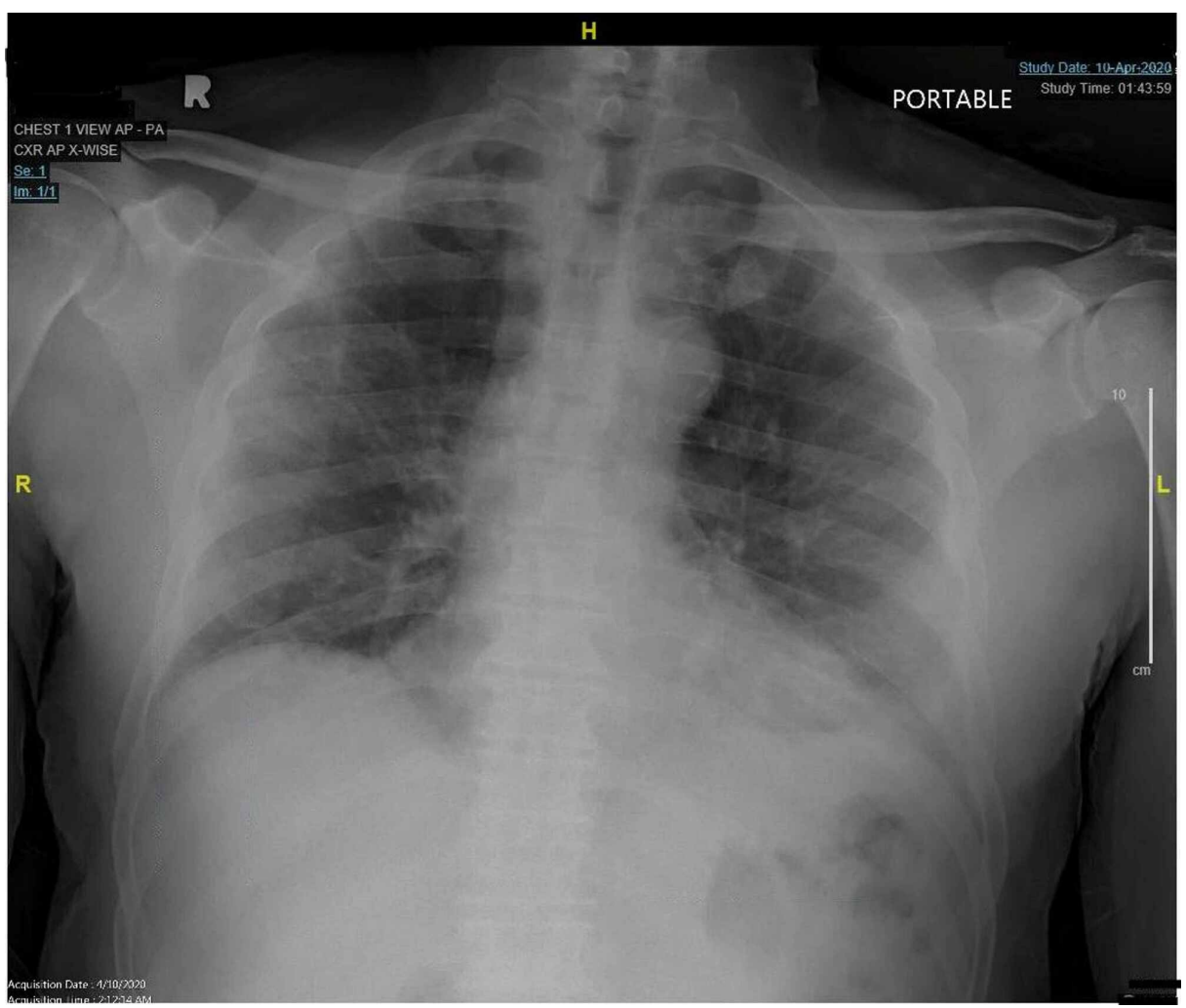

FIGURE 1: Chest $\mathrm{x}$-ray on admission 


\section{Cureus}

The patient was placed on antibiotics (Amoxicillin-Clavulanate, Azithromycin, Hydroxychloroquine and Oseltamivir) as well as supplemental oxygen as needed as per hospital COVID-19 protocol, after which he did have significant improvement in his respiratory status. During the admission, a decline in renal function was noted with rising Blood Urea Nitrogen/ Creatinine (BUN/Cr) levels peaking at $100 \mathrm{mg} / \mathrm{dl}$ and $10.2 \mathrm{mg} / \mathrm{dl}$ respectively, anion gap metabolic acidosis, and hyperkalemia of 6.1. Renal ultrasound at the time, showed a right and left kidney of $11.6 \mathrm{~cm}$ and $11.4 \mathrm{~cm}$ in length respectively, with no hydronephrosis and increased renal cortical echogenicity (Figure 2-4).

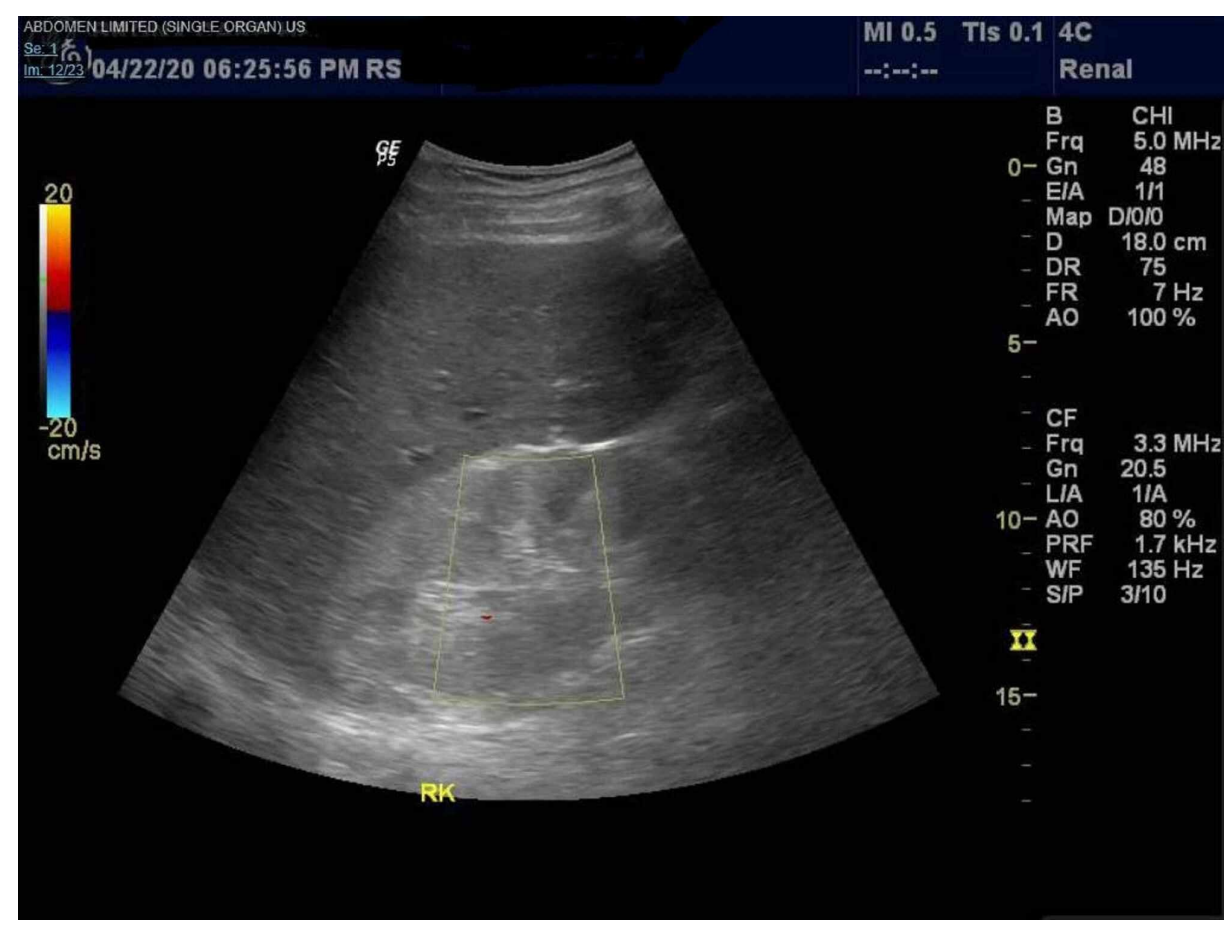

FIGURE 2: Renal Ultrasound

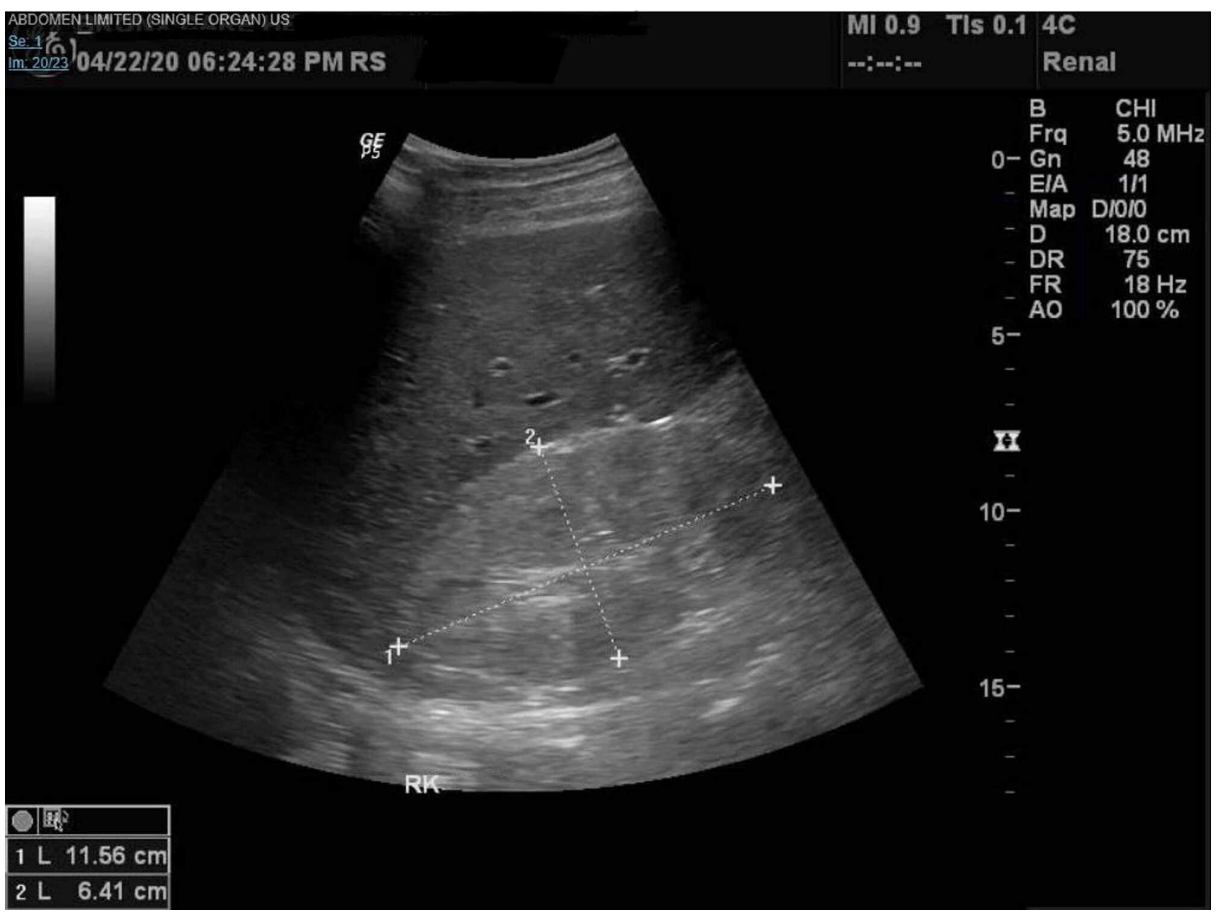

FIGURE 3: Renal Ultrasound - Right kidney 


\section{Cureus}

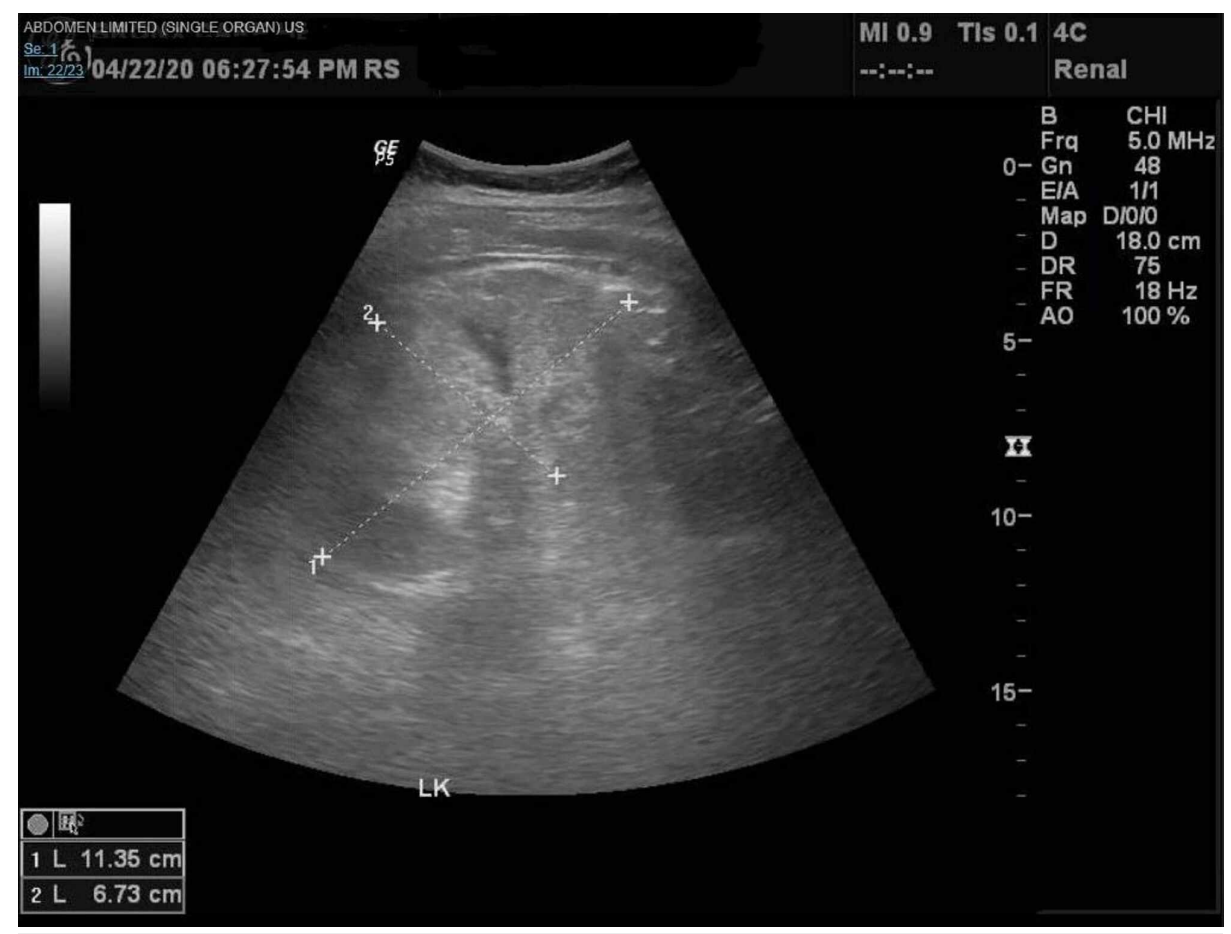

FIGURE 4: Renal Ultrasound - Left kidney

In light of patient's oliguric renal failure and worsening metabolic derangements, the decision was made to start the patient on hemodialysis. Patient's urine output subsequently increased, electrolytes were stable, and serum creatinine was improving off dialysis. Patient was then discharged to complete a 14-day quarantine at home.

About two months later, the patient was readmitted due to a worsening productive cough and associated shortness of breath. Patient complained of worsening dyspnea that was present since the initial diagnosis of COVID 19. Physical examination of cardiac and respiratory systems showed no abnormalities although noted to have +1 bilateral pedal edema. Laboratory values on readmission are presented in Table 2 . Transthoracic echocardiogram showed an ejection fraction within the normal range (56\%) ruling out possible heart failure. Initial lab tests performed also revealed hypoproteinemia, hypoalbuminemia and proteinuria. The urine protein and creatinine ratio confirmed nephrotic range proteinuria of $14865 \mathrm{mg} / \mathrm{g}$. human immunodeficiency virus (HIV) titers, Hepatitis B and C titers, antineutrophil cytoplasmic antibodies (ANCA) vasculitis markers, antinuclear antibody (ANA) markers, and anti-glomerular basement membrane (antiGBM) antibodies were all negative. C3 and C4 level were normal. 


\section{Cureus}

Laboratory Results on readmission

\begin{tabular}{|c|c|c|c|}
\hline WBC Count & $7.4(4.8-10.8 \mathrm{k} / \mathrm{ul})$ & Albumin & $2.4(3.4-4.8 \mathrm{~g} / \mathrm{dl})$ \\
\hline Hemoglobin & $17.9(12.0-16.0 \mathrm{~g} / \mathrm{dl})$ & Bilirubin, Total & $0.0(0.2-1.2 \mathrm{mg} / \mathrm{dL})$ \\
\hline Hematocrit & $52.9(42.0-51.0 \%)$ & Bilirubin, Direct - Conjugated & $0.0(0.0-0.3 \mathrm{mg} / \mathrm{dL})$ \\
\hline Platelet & $221(150-400 \mathrm{k} / \mathrm{ul})$ & Alkaline Phosphatase & 115(56-119 unit/L) \\
\hline Neutro \% & $76.7(40.0-70.0 \%)$ & AST & 19 (9-48 unit/L) \\
\hline \multirow[t]{2}{*}{ Lymphocyte \% } & $16.8(20.0-50.0 \%)$ & ALT & 20 (5-40 unit/L) \\
\hline & & Total Protein & $4.8(6.0-8.5 \mathrm{~g} / \mathrm{dl})$ \\
\hline Sodium & 144 (135-145 mEq/L) & & \\
\hline Potassium & 3.2 (3.5-5.0 mEq/L) & ProBNP & 1330 \\
\hline Bicarbonate & 21 (24-30 mEq/L) & & \\
\hline Chloride & 113 (98-108 mEq/L) & C Reactive Protein & $15.34(<=5.00-\mathrm{mg} / \mathrm{L})$ \\
\hline Glucose & $86(70-120 \mathrm{mg} / \mathrm{dL})$ & & \\
\hline Blood Urea Nitrogen & 33 (8-26 mg/dL) & D-Dimer Assay & $587(0-230 \mathrm{ng} / \mathrm{mL})$ \\
\hline Creatinine & 3.4 (0.5-1.5 mg/dL) & & \\
\hline Calcium & 6.9 (8.5-10.5 mg/dL) & Ferritin & 660 (13-150 ng/mL) \\
\hline
\end{tabular}

\section{TABLE 2: Laboratory Results on Readmission}

WBC- White Blood Cells; AST- Aspartate Aminotransferase; ALT- Alanine aminotransferase; LDH- Lactate Dehydrogenase; proBNP- pro B-type natriuretic peptide

A serum protein electrophoresis (SPEP) was performed and demonstrated monoclonal band proteins confirmed to be immunoglobulin G (IgG) kappa chains on immunofixation and an elevated kappa/lambda ratio of 1.95. A repeat SPEP showed similar results with a worsening kappa/lambda ratio.

A renal biopsy was done to determine the etiology of the nephrotic syndrome with samples outsourced for testing. The patient was managed with calcium channel blockers for blood pressure control and statins. He remained non-oliguric but with slowly worsening serum creatinine levels. Renin-angiotensin-aldosterone system (RAAS) modulation with Angiotensin-converting enzyme (ACE) inhibitors was deferred due to risk of worsening renal function and hyperkalemia and he was discharged to the Nephrology clinic for follow up of biopsy results. Results of the renal biopsy performed ultimately revealed a pattern of collapsing focal segmental glomerulosclerosis (FSGS), in the setting of COVID-19 infection.

Renal biopsy showed collapsing focal segmental glomerulosclerosis, diffuse tubular degenerative changes, tubular atrophy, interstitial fibrosis and moderate interstitial inflammation and moderate to severe arteriosclerosis and arteriolosclerosis. Images from electron microscopy are shown below (Figure 5-9). Subsequent immunofluorescence report can be seen in Table 3. 


\section{Cureus}

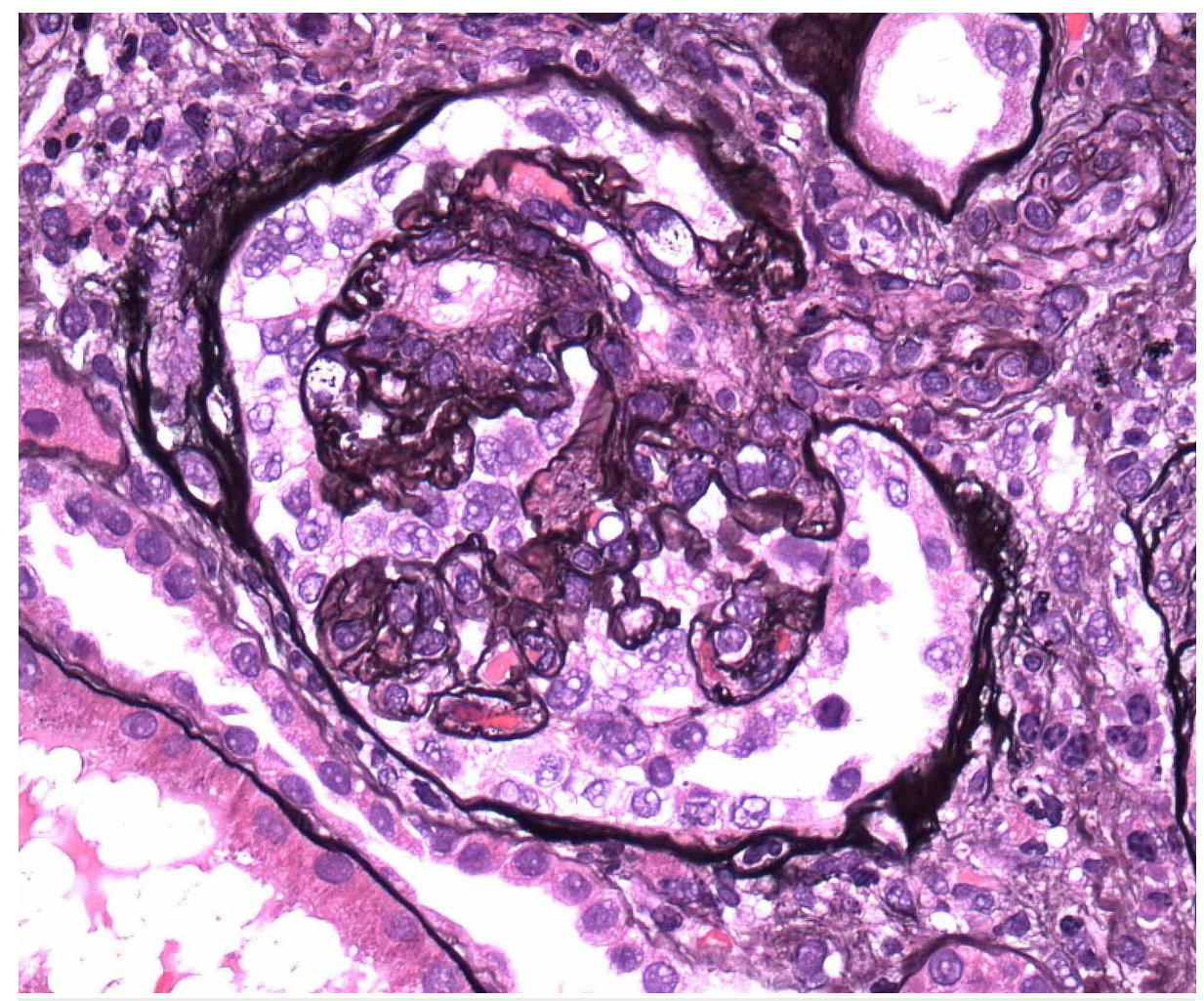

FIGURE 5: Collapsing Focal segmental glomerulosclerosis

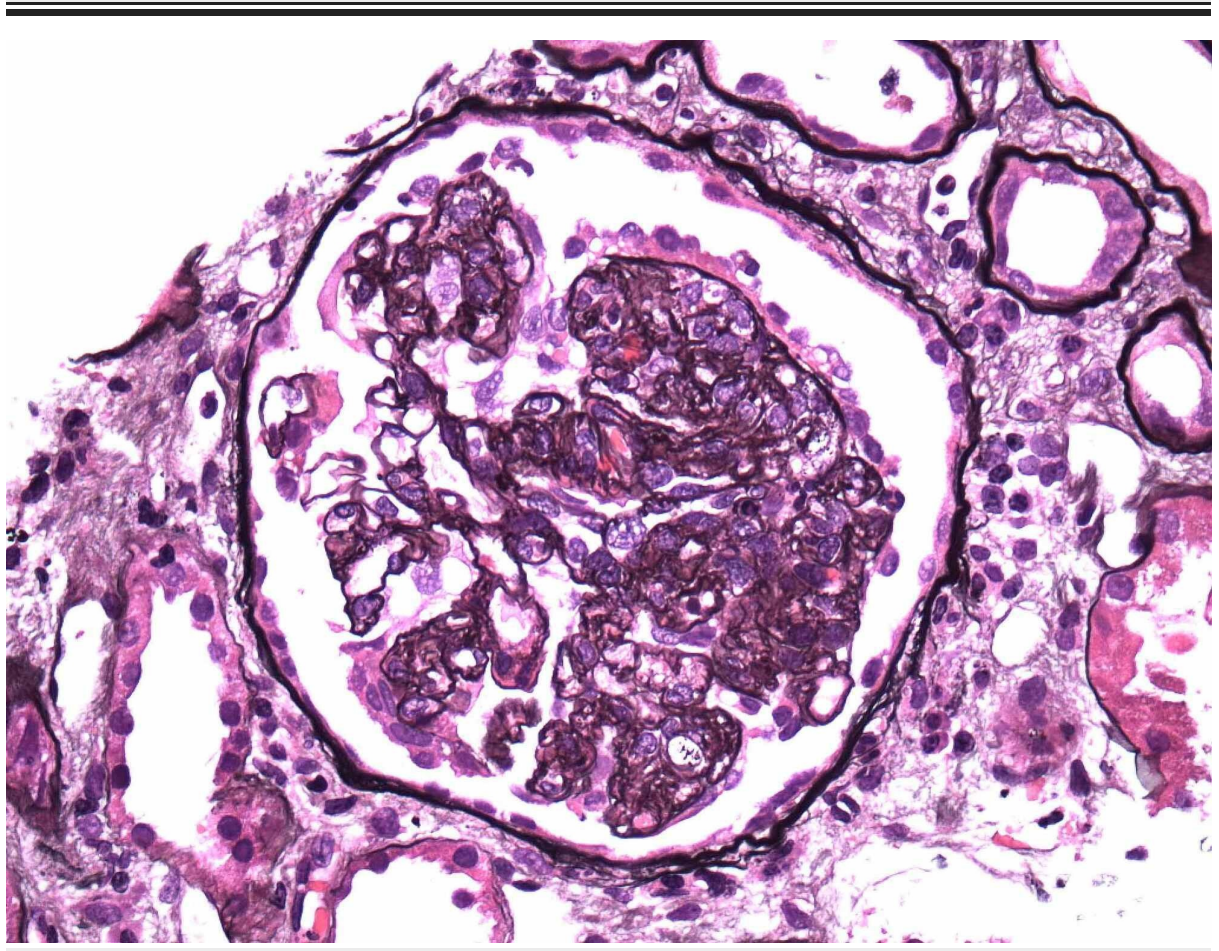

FIGURE 6: Focal segmental glomerulosclerosis 


\section{Cureus}

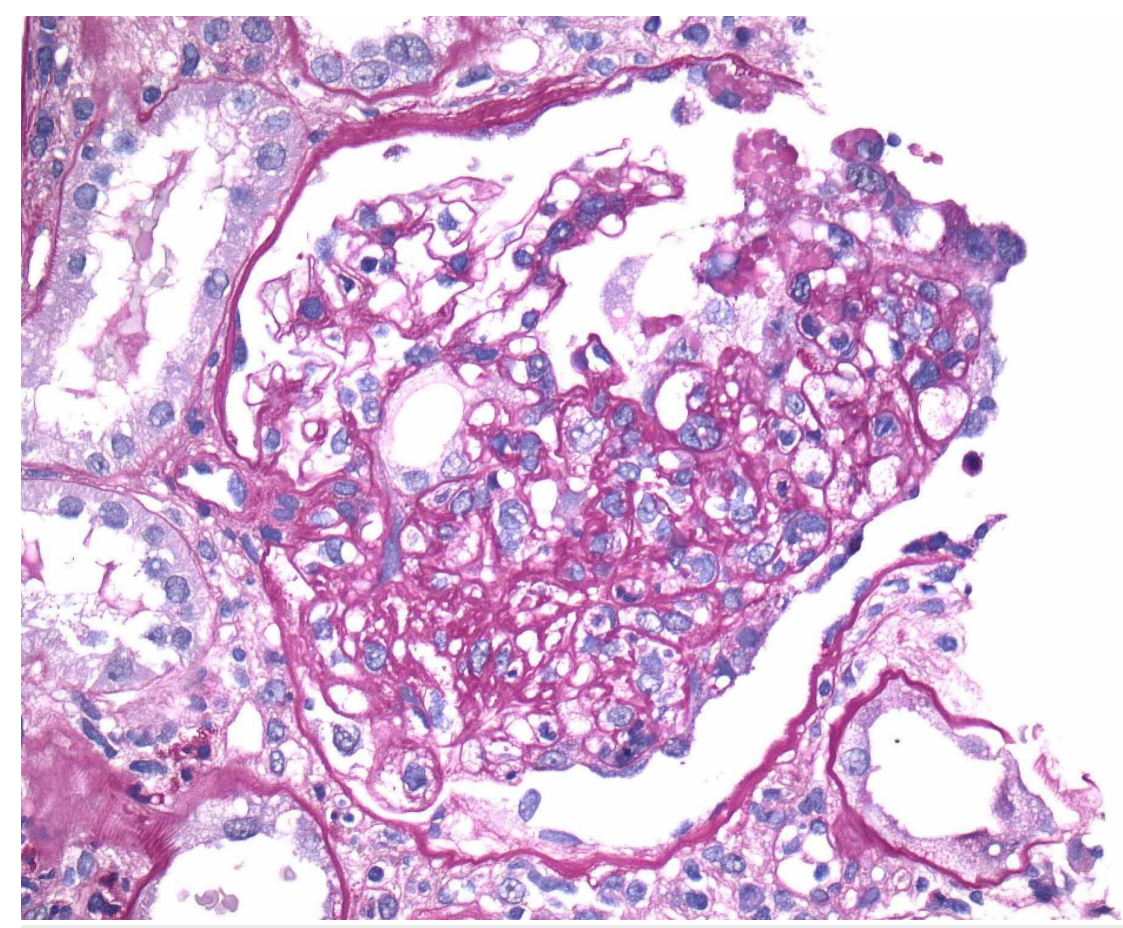

FIGURE 7: Focal segmental glomerulosclerosis

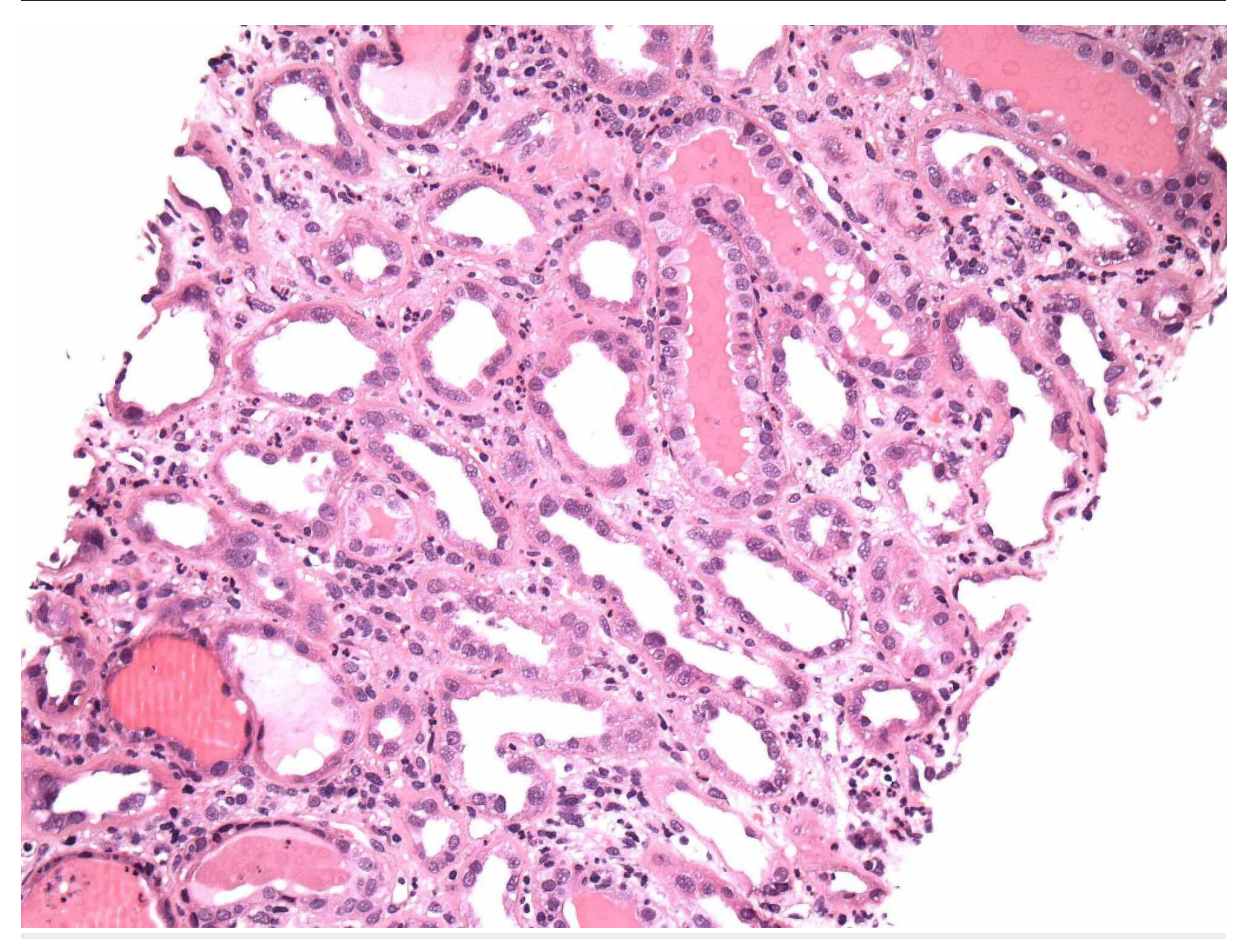

FIGURE 8: Acute Tubular Injury 


\section{Cureus}

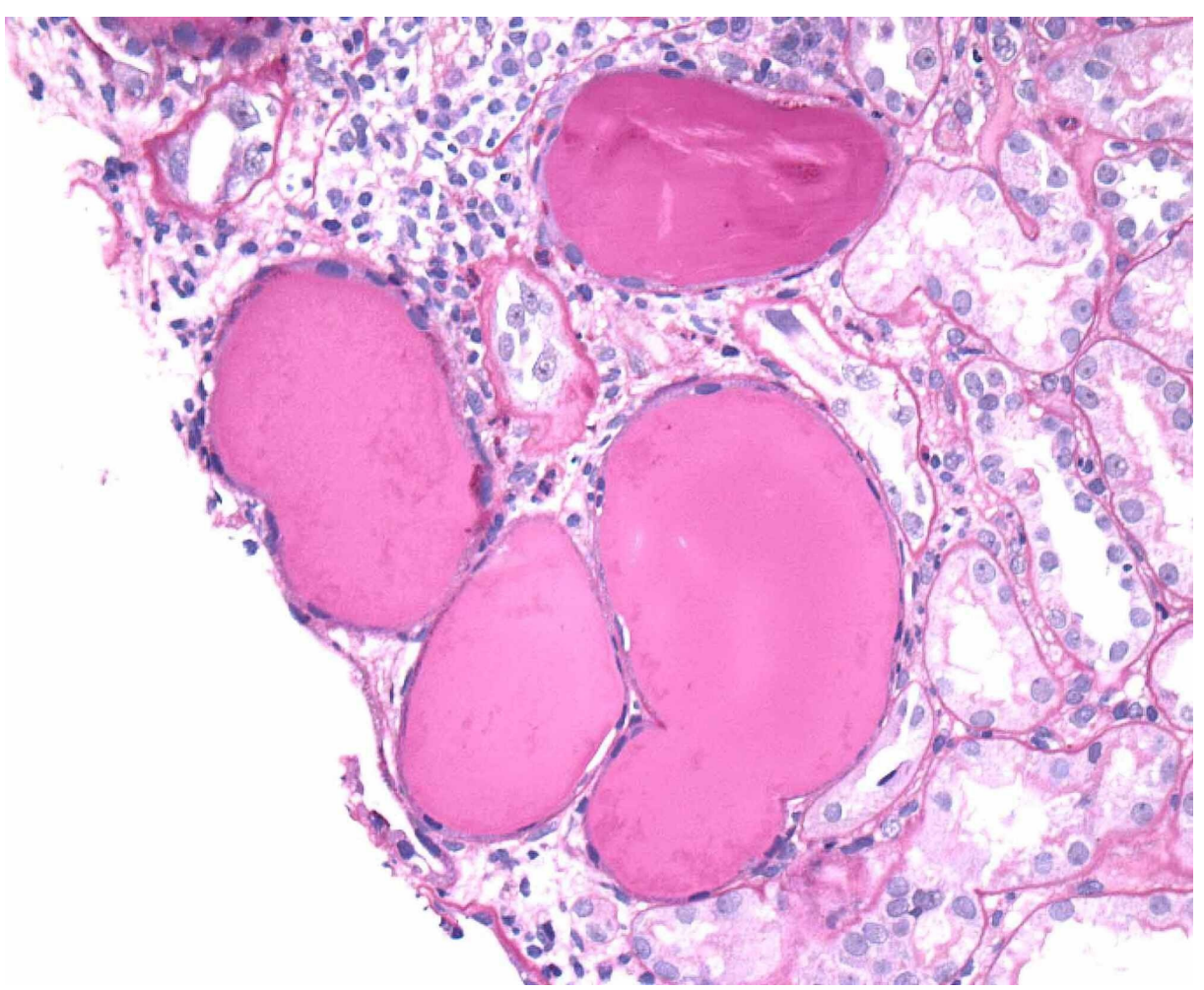

FIGURE 9: Tubular Microcyst

\section{IMMUNOFLUORESCENCE REPORT}

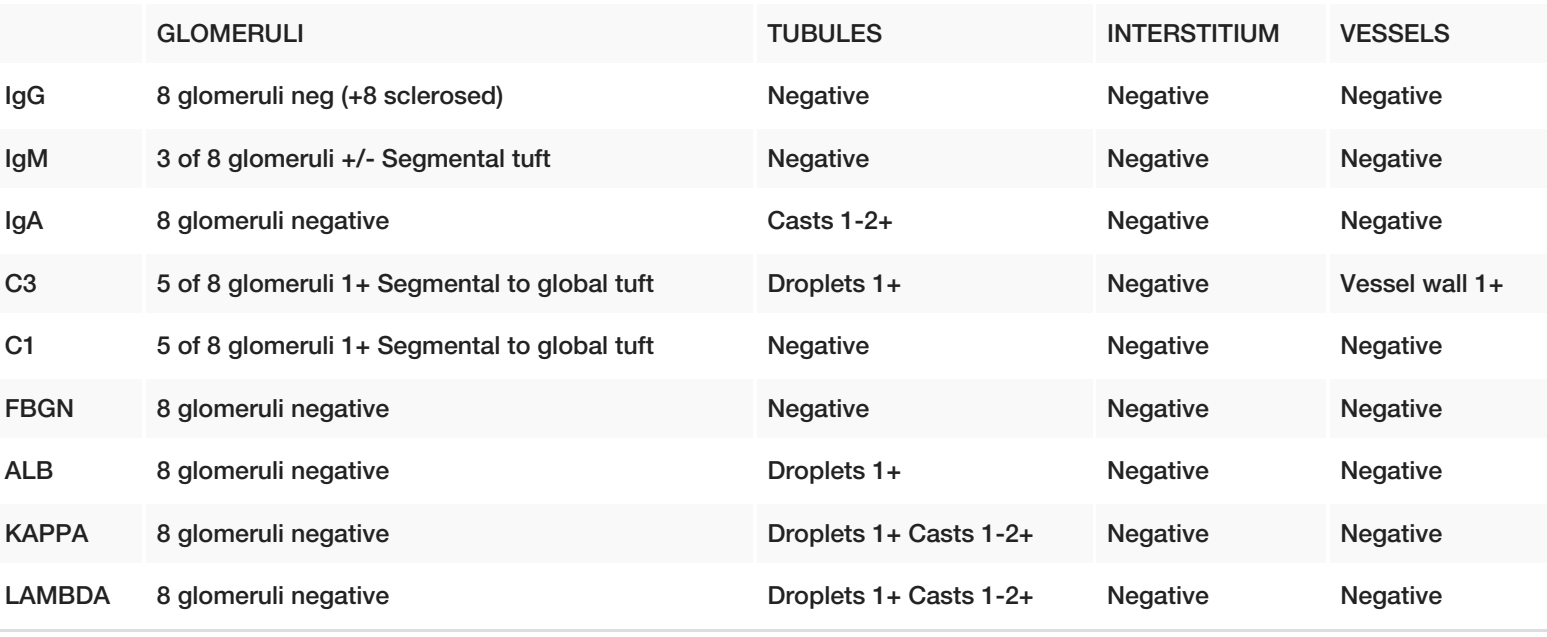

\section{TABLE 3: Immunofluorescence report}

IgG- immunoglobulin G; IgM- immunoglobulin M; IgA- immunoglobulin A; C3- complement component 3; C1- complement component 1; FBGNfibrinogen; ALB- albumin

The patient followed up in the nephrology clinic one month later with elevated blood pressure, however his creatinine and estimated glomerular filtration rate (e-GFR) levels were stable. His blood pressure medications were optimized, but his poor prognosis, likely progression to End-Stage Renal Disease (ESRD) and eventual need for renal replacement therapy was discussed with the patient.

\section{Discussion}

Acute renal failure in coronavirus disease 2019 (COVID-19) infection is associated with higher morbidity, mortality, need for renal replacement therapy and worse overall outcomes. According to one New York 
Hospital, the incidence of acute kidney injury in hospitalized COVID-19 patients was $56.9 \%$. It was also noted that the incidence of acute kidney injury in black patients was $26 \%$ greater than Caucasians [5].

Focal segmental glomerulosclerosis (FSGS) is one of the leading causes of high-grade proteinuria with endstage renal disease (ESRD). There are different histologic variations of FSGS, including classic, collapsing, tip, perihilar, and cellular types. Of all the variants, collapsing FSGS carries the worst prognosis [6]. Collapsing FSGS is associated with a more severe nephrotic syndrome and worse renal outcome than other variants of FSGS. The incidence of ESRD with collapsing FSGS is approximately $73.7 \%$ compared to $28.2 \%$ in non-otherwise specified FSGS. Risk factors with collapsing FSGS, which determine a worse overall outcome include African ancestry, severe proteinuria, lower estimated glomerular filtration rate (eGFR) at baseline [7].

Many viral infections can cause FSGS including human immunodeficiency virus 1 (HIV-1), cytomegalovirus, parvovirus B19 and Epstein-Barr virus $[8,9]$. Histologically, the collapsing variant shows segmental or global mesangial consolidation and loss of endocapillary patency in association with extra-capillary epithelial hypertrophy and/or proliferation [8]. The postulated mechanism of podocyte injury due to viral infections is thought to be via direct podocyte toxicity resulting in dysregulation of the cytoskeleton. In HIV-associated FSGS, there is direct infection of the podocyte that results in this dysregulation and cellular phenotype leading to apoptosis [10]. Additionally, in parvovirus infection with collapsing FSGS, parvovirus has been identified within the podocyte, but it is unclear how parvovirus causes podocyte dysfunction. Acute cytomegalovirus infection has also been associated with collapsing FSGS. It is suggested that acute local renal interferon response is responsible for FSGS lesion rather than the direct effect of the virus on the podocyte [11]. Though the exact mechanism of podocyte injury for COVID-19 infection has not been determined, given the inflammatory milieu involved, it can be postulated that podocyte dysregulation likely occurs due to a cytokine storm. This, in turn, causes a massive release of interferons, granulocyte colonystimulating factor, and interleukins which can cause a direct kidney injury [12]. Though coronavirus was not identified in the podocyte, given the mechanism behind other viral infections, there may be a direct infection of the podocyte causing apoptosis and dysregulation of the cytoskeleton.

According to recent data, the incidence of podocytopathy and proteinuria is higher in patients of African descent $[5,12]$. Studies have shown that the Apolipoprotein L1 (APOL1) gene is expressed exclusively in individuals of African descent. Patients with APOL1 gene have higher baseline proteinuria and kidney disease progression [6]. APOL1 is an under-recognized cause of FSGS leading to ESRD. Approximately 40\% of ESRD is attributed to FSGS in black, of which $72 \%$ is associated with APOL1 genetic variants. Also, the effect of HIV on podocytes is strongest in individuals with two APOL1 risk alleles. The odds of developing HIV associated nephropathy is 29 -fold higher in patients with high-risk alleles [6,8].

Recent studies show that the mechanism of acute kidney injury with SARS-CoV-2 infection is variable and not well understood. Santoriello et al. [13] performed postmortem kidney biopsies on 42 patients and found that acute tubular injury was the main pathologic finding correlating with a history of acute kidney injury. Kudose et al. [12] performed kidney biopsies on 17 patients with COVID 19 infection, of which $29 \%$ had collapsing FSGS as the primary diagnosis for acute kidney injury. Of these 17 patients, $70 \%$ had acute tubular injury seen on pathology.

\section{Conclusions}

In conclusion, collapsing FSGS is an under-recognized cause for renal failure associated with SARS-CoV-2 infection. Acute renal failure with SARS-CoV-2 infection leads to higher mortality and worse prognosis. There should be a low threshold to suspect collapsing FSGS in patients with high-grade proteinuria, acute kidney injury, African descent and nephrotic syndrome in patients with SARS-CoV-2 infection. Additionally, APOL1 genetic testing should be done in patients of African descent with high-grade proteinuria as the progression of renal disease worse in patients with high-risk allele. Further research needs to be done on the mechanism of injury to the podocyte and potential therapies for early intervention.

\section{Additional Information \\ Disclosures}

Human subjects: Consent was obtained by all participants in this study. Conflicts of interest: In compliance with the ICMJE uniform disclosure form, all authors declare the following: Payment/services info: All authors have declared that no financial support was received from any organization for the submitted work. Financial relationships: All authors have declared that they have no financial relationships at present or within the previous three years with any organizations that might have an interest in the submitted work. Other relationships: All authors have declared that there are no other relationships or activities that could appear to have influenced the submitted work.

\section{Acknowledgements}

Madanmohan Patel, MD 


\section{Cureus}

\section{References}

1. Cheng Y, Luo R, Wang K, et al.: Kidney disease is associated with in-hospital death of patients with COVID19. Kidney Int. 2020, 97:829-38. 10.1016/j.kint.2020.03.005

2. D'Agati VD, Alster JM, Jennette JC, et al.: Association of histologic variants in FSGS clinical trial with presenting features and outcomes. Clin J Am Soc Nephrol. 2013, 8:399-406. 10.2215/cjn.06100612

3. Diao B, Feng Z, Wang C, et al.: Human kidney is a target for novel severe acute respiratory syndrome coronavirus 2 (SARS-CoV-2) infection. medRxiv. 2020, 10.1101/2020.03.04.20031120

4. Su H, Yang M, Wan C, et al.: Renal histopathological analysis of 26 postmortem findings of patients with COVID-19 in China. Kidney Int. 2020, 98:219-227. 10.1016/j.kint.2020.04.003

5. Fisher M, Neugarten J, Bellin E, et al.: AKI in hospitalized patients with and without COVID- 19: a comparison study. J Am Soc Nephrol. 2020, 31:2145-57. 10.1681/ASN.2020040509

6. Bose B, Cattran D: Glomerular diseases: FSGS. Clin J Am Soc Nephrol. 2014, 9:626-32. 10.2215/CJN.05810513

7. Laurin L-P, Gasim AM, Derebail VK, et al.: Renal survival in patients with collapsing compared with not otherwise specified FSGS. Clin J Am Soc Nephrol. 2016, 11:1752-59. 10.2215/CJN.13091215

8. Rosenberg A, Kopp JB: Focal Segmental Glomerulosclerosis. Clin J Am Soc Nephrol. 2017, 12:502-517. 10.2215/CJN.05960616

9. $\mathrm{Wu} \mathrm{H}$, Larsen CP, Hernandez-Arroyo CF, et al.: AKI and collapsing glomerulopathy associated with COVID19 and APOL1 high-risk genotype. J Am Soc Nephrol. 2020, 31:1688-95. 10.1681/ASN.2020050558

10. De Vriese AS, Sethi S, Nath KA, et al.: Differentiating primary, genetic, and secondary FSGS in adults: a clinicopathologic approach. J Am Soc Nephrol. 2018, 29:759-74. 10.1681/ASN.2017090958

11. Kupin WL: Viral-associated GN: hepatitis B and other viral infections . Clin J Am Soc Nephrol. 2017, 12:1529-33. 10.2215/CJN.09180816

12. Kudose S, Batal I, Santoriello D, et al.: Kidney biopsy findings in patients with COVID-19 . J Am Soc Nephrol. 2020, 31:1959-68. 10.1681/ASN.2020060802

13. Santoriello D, Khairallah P, Bomback AS, et al.: Postmortem kidney pathology findings in patients with COVID-19. I Am Soc Nephrol. 2020, 31:2158-67. 10.1681/ASN.2020050744 